\title{
Article \\ Anticipated Generalized Backward Doubly Stochastic Differential Equations
}

\author{
Tie Wang $1,2, *$ (i) and Jiaxin $\mathrm{Yu}^{2}$ \\ 1 School of Management, Shanghai University, Shanghai 200444, China \\ 2 School of Mathematics, Liaoning University, Shenyang 110036, China; yjx970622@163.com \\ * Correspondence: wangtie@lnu.edu.cn
}

check for

updates

Citation: Wang, T.; Yu, J. Anticipated Generalized Backward Doubly Stochastic Differential Equations. Symmetry 2022, 14, 114. https:// doi.org/10.3390/sym14010114

Academic Editor: Youssef N. Raffoul

Received: 12 December 2021

Accepted: 7 January 2022

Published: 9 January 2022

Publisher's Note: MDPI stays neutral with regard to jurisdictional claims in published maps and institutional affiliations.

Copyright: (C) 2022 by the authors. Licensee MDPI, Basel, Switzerland. This article is an open access article distributed under the terms and conditions of the Creative Commons Attribution (CC BY) license (https:// creativecommons.org/licenses/by/ $4.0 /)$.

\begin{abstract}
In this paper, we explore a new class of stochastic differential equations called anticipated generalized backward doubly stochastic differential equations (AGBDSDEs), which not only involve two symmetric integrals related to two independent Brownian motions and an integral driven by a continuous increasing process but also include generators depending on the anticipated terms of the solution $(Y, Z)$. Firstly, we prove the existence and uniqueness theorem for AGBDSDEs. Further, two comparison theorems are obtained after finding a new comparison theorem for GBDSDEs.
\end{abstract}

Keywords: anticipated generalized backward doubly stochastic differential equation; existence and uniqueness; comparison theorem

MSC: $60 \mathrm{H} 10 ; 60 \mathrm{H} 30$

\section{Introduction}

Nonlinear backward stochastic differential equations (BSDEs in short) were introduced by Pardoux and Peng [1] in 1990. Since then, BSDEs have been received considerable research attention due to their application in a lot of different research areas, for example, mathematical finance (see El Karoui et al. [2]), stochastic control, differential games and partial differential equations. Ref. [3] proposed a newly optimized symmetric explicit ten-step method with phase-lag of order infinity to numerically solve the Schrodinger equation. Pardoux and Zhang [4] introduced the following equation:

$$
Y_{t}=\xi+\int_{t}^{T} f\left(s, Y_{s}, Z_{s}\right) d s+\int_{t}^{T} h\left(s, Y_{s}\right) d K_{s}-\int_{t}^{T} Z_{s} d W_{s}, t \in[0, T]
$$

where $K_{s}$ is an increasing process, to obtain a probabilistic formula for solutions of semilinear partial differential equations (SPDEs) with a Neumann boundary condition. Ren and Xia [5] further investigated the above topic with reflection, then Ren and Otmani [6] extended this problem to Levy setting.

Pardoux and Peng [7] first presented a class of backward doubly stochastic differential equations (BDSDEs in short) to give a probabilistic representation for a class of quasilinear stochastic partial differential equations. Then Shi et al. [8] gave a comparison theorem for BDSDEs with Lipschitz condition on the coefficients. In this way, Boufoussi et al. [9] gave the following generalized backward doubly stochastic differential equation:

$$
\begin{aligned}
Y_{t}= & +\int_{t}^{T} f\left(s, Y_{s}, Z_{s}\right) d s+\int_{t}^{T} h\left(s, Y_{s}\right) d K_{s}+\int_{t}^{T} g\left(s, Y_{s}, Z_{s}\right) d \overleftarrow{B}_{s} \\
& -\int_{t}^{T} Z_{s} d W_{s}, \quad t \in[0, T],
\end{aligned}
$$

in which the equations not only involve a standard (forward) stochastic Itô integral $d W_{t}$ but also a symmetric backward stochastic Itô integral $d \overleftarrow{B}_{t}$. They first obtained the existence 
and uniqueness for the above equation, then gave the viscosity solution to one kind of semilinear SPDE, a probabilistic representation. Hu and Ren [10] explored this problem with an integral driven by the Levy process. Aman and Mrhardy [11] investigated the Equation (1) with reflection.

Peng and Yang [12] introduced a new type of BSDE called anticipated BSDEs. The generator of these equations includes not only the values of solutions of the present but also the future. The authors found that these anticipated BSDEs have unique solutions under Lipschitz assumptions, a comparison theorem for their solutions, and a duality between them and stochastic differential delay equations. After the work of Peng and Yang [12], Zhang [13] dealt with the comparison theorem of one dimensional anticipated BSDEs under one kind of non-Lipschitz assumption. Xu [14] and zhang [15] introduced the so-called anticipated BDSDES (ABDSDEs). They proved the existence and uniqueness of the solution to these equations, obtained some comparison theorems in the one dimensional case, and studied the duality between ABDSDEs and delayed SDDEs. Reference [16] investigated a coupled system which is composed by a delayed forward doubly stochastic differential equation and an anticipated backward doubly SDE. Recently, Wu et al. [17] proposed the so-called anticipated GBSDEs (AGBSDEs) of the following form:

$$
\left\{\begin{aligned}
& Y_{t}= \xi \\
&+\int_{t}^{T} f\left(s, Y_{s}, Z_{s}, Y_{s+\delta(s)}, Z_{s+\zeta(s)}\right) \mathrm{d} s \\
&+\int_{t}^{T} h\left(s, Y_{s}, Y_{s+\delta(s)}\right) d K_{s}-\int_{t}^{T} Z_{s} \mathrm{~d} W_{s}, t \in[0, T] \\
& Y_{t}=\xi_{t}, \quad Z_{t}=\eta_{t}, \quad t \in[T, T+K]
\end{aligned}\right.
$$

where $\delta(\cdot)$ and $\zeta(\cdot)$ are given $\mathbb{R}^{+}$-valued continuous functions and for $\phi(\cdot)=\delta(\cdot), \zeta(\cdot)$ such that:

(A1) there exists a constant $K \geq 0$ such that, for all $t \in[0, T], t+\phi(t) \leqslant T+K$;

(A2) there exists a constant $M \geqslant 0$ such that, for all $t \in[0, T]$ and for all non-negative and integrable $g(\cdot)$,

$$
\int_{t}^{T} g(s+\phi(s)) \mathrm{d} s \leqslant M \int_{t}^{T+K} g(s) \mathrm{d} s, \int_{t}^{T} g(s+\phi(s)) d K_{s} \leqslant M \int_{t}^{T+K} g(s) d K_{s},
$$

and for any interval $[\alpha, \beta],[\alpha+u, \beta+u] \in[0, T+K], u>0$, we have $\mathrm{d} K_{s}([\alpha, \beta]) \leq \mathrm{d} K_{s}([\alpha+$ $u, \beta+u])$, where $\mathrm{d} K_{s}$ is a measure generated by $K$ on $[0, T+K]$.

In this paper, we are concerned with the following anticipated GBDSDEs:

$$
\left\{\begin{aligned}
Y_{t}= & Y_{T}+\int_{t}^{T} f\left(s, Y_{s}, Z_{s}, Y_{s+\delta_{1}(s)}, Z_{s+\zeta(s)}\right) \mathrm{d} s+\int_{t}^{T} h\left(s, Y_{s}, Y_{s+\delta_{2}(s)}\right) d K_{s} \\
& +\int_{t}^{T} g\left(s, Y_{s}, Z_{s}, Y_{s+\delta_{3}(s)}, Z_{s+\bar{\zeta}(s)}\right) \mathrm{d} \overleftarrow{B}_{s}-\int_{t}^{T} Z_{s} \mathrm{~d} W_{s}, \quad t \in[0, T], \\
Y_{t}= & \xi_{t}, \quad Z_{t}=\eta_{t}, \quad t \in[T, T+K],
\end{aligned}\right.
$$

where $\delta_{i}(\cdot), i=1,2,3, \zeta(\cdot)$ and $\bar{\zeta}(\cdot)$ are given $\mathbb{R}^{+}$-valued continuous functions such that (A1) and (A2). We will prove that the solution of the above AGBDSDE exists uniquely under proper assumptions, and two versions of one dimensional comparison theorems are given. These results are the cornerstones of AGBDSDEs applied to the obstacle problem for some SPDEs with the nonlinear Neumann boundary condition and some stochastic control problems with delay.

The organization of this paper is as follows. In Section 2, some preliminaries, assumptions and definitions are given. In Section 3, we focus on the existence and uniqueness of the solutions of anticipated GBDSDEs. In Section 4, two comparison theorems are given, and in the last section, the conclusion and future work are presented. 


\section{Preliminaries}

Throughout the paper, we use $|x|$ and $\|A\|=\sqrt{\operatorname{Tr}\left(A A^{*}\right)}$ to denote the norm of a vector $x \in \mathbb{R}^{k}$ and a matrix $A \in k \times d$, respectively, where $A^{*}$ is the transpose of $A$. Let $\left\{W_{t} ; 0 \leq t \leq T\right\}$ and $\left\{B_{t} ; 0 \leq t \leq T\right\}$ be two mutually independent standard Browning motions, with values respectively in $\mathbb{R}^{d}$ and $\mathbb{R}^{l}$ on a complete probability space $(\Omega, \mathcal{F}, \mathbb{P})$. Let $T>0, K \geq 0$ be fixed constants. Let $\mathcal{N}$ denote the class of $P$-null sets of $\mathcal{F}$. For any $t \in[0, T+K]$, we define:

$$
\mathcal{F}_{t} \triangleq \mathcal{F}_{0, t}^{W} \vee \mathcal{F}_{t, T+K}^{B}
$$

where for any processes $\left\{\eta_{t}\right\}, \mathcal{F}_{s, t}^{\eta}=\sigma\left\{\eta_{r}-\eta_{s} ; s \leq r \leq t\right\} \vee \mathcal{N}$. It is worth noting that $\left\{\mathcal{F}_{t}, t \in[0, T+K]\right\}$ is not a filtration because $\left\{\mathcal{F}_{0, t}^{W}, t \in[0, T+K]\right\}$ is increasing and $\left\{\mathcal{F}_{t, T}^{B}, t \in[0, T+K]\right\}$ is decreasing. Let $K_{t}$ be a continuous, increasing and $\mathcal{F}_{t}$-adapted process on $[0, T+K]$ with $K_{0}=0$. We will use the following notations: for any $n \in \mathbb{N}$,

(i) $\mathcal{M}^{2}\left(0, T ; \mathbb{R}^{n}\right) \triangleq\left\{\varphi: \Omega \times[0, T] \rightarrow \mathbb{R}^{n} \mid \varphi\right.$ is a $\mathcal{F}_{t}$-progressively measurable processes such that $\left.\|\varphi\|_{\mathcal{M}^{2}}^{2}=\mathbb{E}\left(\int_{0}^{T}\left|\varphi_{t}\right|^{2} d t\right)<\infty\right\}$;

(ii) $\mathcal{S}^{2}\left([0, T] ; \mathbb{R}^{n}\right) \triangleq\left\{\varphi: \Omega \times[0, T] \rightarrow \mathbb{R}^{n} \mid \varphi\right.$ is a continuous and $\mathcal{F}_{t^{-}}$progressively measurable processes such that $\|\varphi\|_{\mathcal{S}^{2}}^{2}=\mathbb{E}\left(\sup _{0 \leq t \leq T}\left|\varphi_{t}\right|^{2}\right)<\infty$;

(iii) $L^{2}\left(\mathcal{F}_{T} ; \mathbb{R}^{n}\right) \triangleq\left\{\xi: \xi \in \mathbb{R}^{n} \mid \xi\right.$ is a $\mathcal{F}_{T}$-measurable random variable with $\left.\mathbb{E}|\xi|^{2}<\infty\right\}$.

Let $f(t, \cdot, \cdot, \cdot, \cdot, \cdot): \Omega \times \mathbb{R}^{k} \times \mathbb{R}^{k \times d} \times \mathcal{M}^{2}\left(t, T+K ; \mathbb{R}^{k}\right) \times \mathcal{M}^{2}\left(t, T+K ; \mathbb{R}^{k \times d}\right) \rightarrow L^{2}\left(\mathcal{F}_{t} ;\right.$ $\left.\mathbb{R}^{k}\right), g(t, \cdot, \cdot, \cdot, \cdot, \cdot): \Omega \times \mathbb{R}^{k} \times \mathbb{R}^{k \times d} \times \mathcal{M}^{2}\left(t, T+K ; \mathbb{R}^{k}\right) \times \mathcal{M}^{2}\left(t, T+K ; \mathbb{R}^{k \times d}\right) \rightarrow L^{2}\left(\mathcal{F}_{t} ;\right.$ $\left.\mathbb{R}^{k \times l}\right), h(t, \cdot, \cdot, \cdot): \Omega \times \mathbb{R}^{k} \times \mathcal{M}^{2}\left(t, T+K ; \mathbb{R}^{k}\right) \rightarrow L^{2}\left(\mathcal{F}_{t} ; \mathbb{R}^{k}\right)$. We make the following assumptions about $(\xi, f, g, h)$ :

Hypothesis 1 (H1). For $\xi \in S^{2}\left([T, T+K] ; \mathbb{R}^{k}\right), \eta \in \mathcal{M}^{2}\left(T, T+K ; \mathbb{R}^{k \times d}\right)$, we assume for each $\mu \in R^{+}$,

$$
\mathbb{E}\left[\sup _{t \in[T, T+K]} e^{\mu K_{t}}\left|\xi_{t}\right|^{2}\right]<\infty, \mathbb{E}\left[\int_{T}^{T+K} e^{\mu K_{t}}\left|\xi_{t}\right|^{2} d K_{t}\right]<\infty, \mathbb{E}\left[\int_{T}^{T+K} e^{\mu K_{t}}\left\|\eta_{t}\right\|^{2} d t\right]<\infty .
$$

Hypothesis 2 (H2). For all $s \in[0, T], y, y^{\prime} \in \mathbb{R}^{k}, z, z^{\prime} \in \mathbb{R}^{k \times d}, \theta(\cdot), \theta^{\prime}(\cdot) \in \mathcal{M}^{2}\left(s, T+K ; \mathbb{R}^{k}\right)$, $\vartheta(\cdot), \vartheta^{\prime}(\cdot) \in \mathcal{M}^{2}\left(s, T+K ; \mathbb{R}^{k \times d}\right), r, \bar{r} \in[s, T+K]$, we have

$$
\left\{\begin{array}{l}
\left|f(s, y, z, \theta(r), \vartheta(\bar{r}))-f\left(s, y^{\prime}, z^{\prime}, \theta^{\prime}(r), \vartheta^{\prime}(\bar{r})\right)\right|^{2} \\
\leqslant C\left(\left|y-y^{\prime}\right|^{2}+\left\|z-z^{\prime}\right\|^{2}+\mathbb{E}^{\mathcal{F}_{s}}\left[\left|\theta(r)-\theta^{\prime}(r)\right|^{2}\right]+\mathbb{E}^{\mathcal{F}_{s}}\left[\left\|\vartheta(\bar{r})-\vartheta^{\prime}(\bar{r})\right\|^{2}\right]\right) \\
\left\|g(s, y, z, \theta(r), \vartheta(\bar{r}))-g\left(s, y^{\prime}, z^{\prime}, \theta^{\prime}(r), \vartheta^{\prime}(\bar{r})\right)\right\|^{2} \\
\leqslant C\left(\left|y-y^{\prime}\right|^{2}+\mathbb{E}^{\mathcal{F}_{s}}\left[\left|\theta(r)-\theta^{\prime}(r)\right|^{2}\right]\right)+\alpha_{1}\left\|z-z^{\prime}\right\|^{2}+\alpha_{2} \mathbb{E}^{\mathcal{F}_{s}}\left[\left\|\vartheta(\bar{r})-\vartheta^{\prime}(\bar{r})\right\|^{2}\right] \\
\left\langle y-y^{\prime}, h(s, y, \theta(r))-f\left(s, y^{\prime}, \theta^{\prime}(r)\right)\right\rangle \\
\leqslant \bar{C}\left|y-y^{\prime}\right|^{2}+\beta\left|y-y^{\prime}\right| \mathbb{E}^{\mathcal{F}_{s}}\left[\left|\theta(r)-\theta^{\prime}(r)\right|\right]
\end{array}\right.
$$

where $C>0, \bar{C}<0, \beta>0,0<\alpha_{1}<1,0<\alpha_{1}+\alpha_{2} M<1$ are five constants.

Hypothesis 3 (H3). For any $s \in[0, T], y \in \mathbb{R}^{k}, z \in \mathbb{R}^{k \times d}, \theta(\cdot) \in \mathcal{M}^{2}\left(s, T+K ; \mathbb{R}^{k}\right), \vartheta(\cdot) \in$ $\mathcal{M}^{2}\left(s, T+K ; \mathbb{R}^{k \times d}\right), r, \bar{r} \in[s, T+K], \mu \in R^{+}$, we have

$$
\left\{\begin{array}{l}
|f(s, y, z, \theta(r), \vartheta(\bar{r}))| \leqslant \psi_{1}(s)+K\left(|y|+\|z\|+\mathbb{E}^{\mathcal{F}_{s}}[|\theta(r)|]+\mathbb{E}^{\mathcal{F}_{s}}[\|\vartheta(\bar{r})\|]\right), \\
\|g(s, y, z, \theta(r), \vartheta(\bar{r}))\| \leqslant \psi_{2}(s)+K\left(|y|+\|z\|+\mathbb{E}^{\mathcal{F}_{s}}[|\theta(r)|]+\mathbb{E}^{\mathcal{F}_{s}}[\|\vartheta(\bar{r})\|]\right), \\
|h(s, y, \theta(r))| \leqslant \psi_{3}(s)+K\left(|y|+\mathbb{E}^{\mathcal{F}_{s}}[|\theta(r)|]\right), \\
\mathbb{E}\left(\int_{0}^{T} e^{\mu K_{s}}\left|\psi_{1}(s)\right|^{2} d s+\int_{0}^{T} e^{\mu K_{s}}\left|\psi_{2}(s)\right|^{2} d s+\int_{0}^{T} e^{\mu K_{t}}\left|\psi_{3}(s)\right|^{2} K_{s}\right)<\infty,
\end{array}\right.
$$


where $\psi_{i}, i=1,2,3$ are three adapted processes with values in $[1,+\infty)$ and $K>0$ is a constant. The first and second inequalities on the above are the Lipschitze conditions for $f$ and $g$ with anticipated terms, respectively.

Definition 1. A solution for AGDSDE is a pair $\left(Y_{t}, Z_{t}\right) \in S^{2}\left([0, T+K] ; \mathbb{R}^{n}\right) \times \mathcal{M}^{2}(0, T+$ $K ; \mathbb{R}^{n \times d}$ ) such that for any $0 \leq t \leq T$,

$$
\left\{\begin{aligned}
Y_{t}= & \xi_{T}+\int_{t}^{T} f\left(s, Y_{s}, Z_{s}, Y_{s+\delta_{1}(s)}, Z_{s+\zeta(s)}\right) \mathrm{d} s+\int_{t}^{T} h\left(s, Y_{s}, Y_{s+\delta_{2}(s)}\right) d K_{s} \\
& +\int_{t}^{T} g\left(s, Y_{s}, Z_{s}, Y_{s+\delta_{3}(s)}, Z_{s+\bar{\zeta}(s)}\right) \mathrm{d} \overleftarrow{B}_{s}-\int_{t}^{T} Z_{s} \mathrm{~d} W_{s}, \quad t \in[0, T] \\
Y_{t}= & \xi_{t}, \quad Z_{t}=\eta_{t}, \quad t \in[T, T+K] .
\end{aligned}\right.
$$

\section{Existence and Uniqueness Theorem}

In order to obtain the existence and uniqueness result, we need the following two priori estimates.

Proposition 1. Assume that (A1), (A2), (H1), (H2) and (H3) hold. Ff $\left\{\left(Y_{t}, Z_{t}\right) ; 0 \leq t \leq T+K\right\}$ is a solution of $A G B D S D E$ (2) and $\mu>0, \lambda>\max \{\beta(1+M)-|\bar{C}|, 0\}$, we get:

$$
\begin{aligned}
& \mathbb{E}\left[\sup _{t \in[0, T]} e^{\mu t+\lambda K_{t}}\left|Y_{t}\right|^{2}+\int_{0}^{T} e^{\mu s+\lambda K_{s}}\left\|Z_{s}\right\|^{2} d s+\int_{0}^{T} e^{\mu s+\lambda K_{s}}\left|Y_{s}\right|^{2} d K_{s}\right] \leq \widetilde{C} \mathbb{E}\left(\sup _{T \leq t \leq T+K} e^{\mu t+\lambda K_{t}}\left|\xi_{t}\right|^{2}\right. \\
& +\int_{T}^{T+K} e^{\mu s+\lambda K_{s}}\left|\xi_{s}\right|^{2} d s+\int_{T}^{T+K} e^{\mu s+\lambda K_{s}}\left\|\eta_{s}\right\|^{2} d s+\int_{T}^{T+K} e^{\mu s+\lambda K_{s}}\left|\xi_{s}\right|^{2} d K_{s} \\
& \left.+\int_{0}^{T} e^{\mu s+\lambda K_{s}}\left|\psi_{1}(s)\right|^{2} d s+\int_{0}^{T} e^{\mu s+\lambda K_{s}}\left|\psi_{2}(s)\right|^{2} d s+\int_{0}^{T} e^{\mu s+\lambda K_{s}}\left|\psi_{3}(s)\right|^{2} d K_{s}\right],
\end{aligned}
$$

where $\widetilde{C}$ is a constant.

Proof. In the following, we assume $K_{T+K}$ is a bounded random variable, and then apply Fatou's lemma to obtain the general result. From Itô's formula, we have:

$$
\begin{aligned}
& e^{\mu t+\lambda K_{t}}\left|Y_{t}\right|^{2}+\int_{t}^{T} e^{\mu s+\lambda K_{s}}\left\|Z_{s}\right\|^{2} d s+\lambda \int_{t}^{T} e^{\mu s+\lambda K_{s}}\left|Y_{s}\right|^{2} d K_{s}=e^{\mu T+\lambda K_{T}}\left|\xi_{T}\right|^{2} \\
& +2 \int_{t}^{T} e^{\mu s+\lambda K_{s}}\left\langle Y_{s}, f\left(s, Y_{s}, Z_{s}, Y_{s+\delta_{1}(s)}, Z_{s+\zeta(s)}\right)\right\rangle d s+2 \int_{t}^{T} e^{\mu s+\lambda K_{s}}\left\langle Y_{s}, h\left(s, Y_{s}, Y_{s+\delta_{2}(s)}\right)\right\rangle d K_{s} \\
& +2 \int_{t}^{T} e^{\mu s+\lambda K_{s}}\left\langle Y_{s}, g\left(s, Y_{s}, Z_{s}, Y_{s+\delta_{3}(s)}, Z_{s+\bar{\zeta}(s)}\right) \mathrm{d} \overleftarrow{B}_{s}\right\rangle-2 \int_{t}^{T} e^{\mu s+\lambda K_{s}}\left\langle Y_{s}, Z_{s} \mathrm{~d} W_{s}\right\rangle \\
& -\mu \int_{t}^{T} e^{\mu s+\lambda K_{s}}\left|Y_{s}\right|^{2} d s+\int_{t}^{T} e^{\mu s+\lambda K_{s}}\left\|g\left(s, Y_{s}, Z_{s}, Y_{s+\delta_{3}(s)}, Z_{s+\bar{\zeta}(s)}\right)\right\|^{2} d s .
\end{aligned}
$$

According to the assumptions (H2), (H3) and Yong's inequality, for any $\theta>0$, we get:

$$
\begin{aligned}
2\left\langle Y_{s}, f\left(s, Y_{s}, Z_{s}, Y_{s+\delta_{1}(s)}, Z_{s+\zeta(s)}\right)\right\rangle \leq & \theta\left|Y_{s}\right|^{2}+\frac{2 C}{\theta}\left(\left|Y_{s}\right|^{2}+\left|Z_{s}\right|^{2}+\mathbb{E}^{\mathscr{F}_{s}}\left|Y_{s+\delta_{1}(s)}\right|^{2}+\mathbb{E}^{\mathscr{F}_{s}}\left|Z_{s+\zeta(s)}\right|^{2}\right) \\
& +\frac{2}{\theta}\left|\psi_{1}(s)\right|^{2}, \\
2\left\langle Y_{s}, h\left(s, Y_{s}, Y_{s+\delta_{2}(s)}\right)\right\rangle \leq & 2 \bar{C}\left|Y_{s}\right|^{2}+2 \beta\left|Y_{s}\right| \mathbb{E}^{\mathscr{F}_{s}}\left|Y_{s+\delta_{2}(s)}\right|+2\left|Y_{s}\right|\left|\psi_{3}(t)\right| \\
\leq & (2 \bar{C}+|\bar{C}|+\beta)\left|Y_{s}\right|^{2}+\beta \mathbb{E}^{\mathscr{F}_{s}}\left|Y_{s+\delta_{2}(s)}\right|^{2}+\frac{1}{|\bar{C}|}\left|\psi_{3}(s)\right|^{2}, \\
\left\|g\left(s, Y_{s}, Z_{s}, Y_{s+\delta_{3}(s)}, Z_{s+\bar{\zeta}(s)}\right)\right\|^{2} \leq & \left(1+\frac{1}{\theta}\right)\left(C\left(\left|Y_{s}\right|^{2}+\mathbb{E}^{\mathscr{F}_{s}}\left|Y_{s+\delta_{3}(s)}\right|^{2}\right)+\alpha_{1}|| Z_{s} \|^{2}+\alpha_{2} \mathbb{E}^{\mathscr{F}_{s}}\left|Z_{s+\bar{\zeta}(s)}\right|^{2}\right) \\
& +(1+\theta)\left|\psi_{2}(s)\right|^{2} .
\end{aligned}
$$


Consequently, we have:

$$
\begin{aligned}
& E\left[e^{\mu t+\lambda K_{t}}\left|Y_{t}\right|^{2}+\left(1-\alpha_{1}-\alpha_{2} M-\frac{2 C(1+M)+\alpha_{1}+\alpha_{2} M}{\theta}\right) \int_{t}^{T} e^{\mu s+\lambda K_{s}}\left\|Z_{s}\right\|^{2} d s\right. \\
& \left.+(\lambda+|\bar{C}|-\beta(1+M)) \int_{t}^{T} e^{\mu s+\lambda K_{s}}\left|Y_{s}\right|^{2} d K_{s}\right] \leq E\left[e^{\mu T+\lambda K_{T}}\left|\xi_{T}\right|^{2}\right. \\
& +\left(\theta+\frac{3 C(1+M)}{\theta}+C(1+M)-\mu\right) \int_{t}^{T} e^{\mu s+\lambda K_{s}}\left|Y_{s}\right|^{2} d s+\left(\frac{3 C M}{\theta}+C M\right) \int_{T}^{T+K} e^{\mu s+\lambda K_{s}}\left|\xi_{s}\right|^{2} d s \\
& +\left(\frac{2 C M}{\theta}+\left(1+\frac{1}{\theta}\right) \alpha_{2} M\right) \int_{T}^{T+K} e^{\mu s+\lambda K_{s}}\left\|\eta_{s}\right\|^{2} d s+\frac{2}{\theta} \int_{t}^{T} e^{\mu s+\lambda K_{s}}\left|\psi_{1}(s)\right|^{2} d s \\
& \left.+\beta M \int_{T}^{T+K} e^{\mu s+\lambda K_{s}}\left|\xi_{s}\right|^{2} d K_{s}+\frac{1}{|\bar{C}|} \int_{t}^{T} e^{\mu s+\lambda K_{s}}\left|\psi_{3}(s)\right|^{2} d K_{s}+(1+\theta) \int_{t}^{T} e^{\mu s+\lambda K_{s}}\left|\psi_{2}(s)\right|^{2} d s\right] .
\end{aligned}
$$

Thus, choosing $\theta=\frac{2 C(1+M)+\alpha_{1}+\alpha_{2} M}{1-\alpha_{1}-\alpha_{2} M}+|\mu|+1$, and from Gronwall's inequality, we can obtain:

$\sup _{t \in[0, T]} \mathbb{E}\left[e^{\mu t+\lambda K_{t}}\left|Y_{t}\right|^{2}+\int_{0}^{T} e^{\mu s+\lambda K_{s}}\left\|Z_{s}\right\|^{2} d s+\int_{0}^{T} e^{\mu s+\lambda K_{s}}\left|Y_{s}\right|^{2} d K_{s}\right] \leq \hat{C} \mathbb{E}\left(\sup _{T \leq t \leq T+K} e^{\mu t+\lambda K_{t}}\left|\xi_{t}\right|^{2}\right.$

$$
\begin{aligned}
& +\int_{T}^{T+K} e^{\mu s+\lambda K_{s}}\left|\xi_{s}\right|^{2} d s+\int_{T}^{T+K} e^{\mu s+\lambda K_{s}}\left\|\eta_{s}\right\|^{2} d s+\int_{T}^{T+K} e^{\mu s+\lambda K_{s}}\left|\xi_{s}\right|^{2} d K_{s} \\
& \left.+\int_{0}^{T} e^{\mu s+\lambda K_{s}}\left|\psi_{1}(s)\right|^{2} d s+\int_{0}^{T} e^{\mu s+\lambda K_{s}}\left|\psi_{2}(s)\right|^{2} d s+\int_{0}^{T} e^{\mu s+\lambda K_{s}}\left|\psi_{3}(s)\right|^{2} d K_{s}\right] .
\end{aligned}
$$

Using the Burkholder-Davis-Gundy's and above inequality, the desired result follows.

Proposition 2. Denote $(\bar{Y}, \bar{Z}, \bar{\xi}, \bar{\eta}, \bar{f}, \bar{g}, \bar{h}, \bar{K})=\left(Y-Y^{\prime}, Z-Z^{\prime}, \xi-\xi^{\prime}, \eta-\eta^{\prime}, f-f^{\prime}, g-\right.$ $\left.g^{\prime}, K-K^{\prime}\right)$, then, for any $\mu>|2 \bar{C}+\beta(1+M)|$, there exists a constant $\widetilde{C}>0$ such that:

$$
\begin{aligned}
& \mathbb{E}\left[\sup _{t \in[0, T]} e^{\mu A_{t}}\left|\bar{Y}_{t}\right|^{2}+\int_{0}^{T} e^{\mu A_{s}}\left\|\bar{Z}_{s}\right\|^{2} d s\right] \leq \widetilde{C} \mathbb{E}\left[e^{\mu K_{T}}\left|\bar{\xi}_{T}\right|^{2}\right. \\
& +\int_{T}^{T+K} e^{\mu A_{s}}\left\|\bar{\eta}_{s}\right\|^{2} d s+\int_{T}^{T+K} e^{\mu A_{s}}\left|\bar{\zeta}_{s}\right|^{2} d K_{s}^{\prime}+\int_{0}^{T} e^{\mu A_{s}}\left|h\left(s, Y_{s}, Y_{s+\delta_{2}(s)}\right)\right|^{2}\|\bar{K}\|_{s} \\
& +\int_{0}^{T} e^{\mu A_{s}}\left|f\left(s, Y_{s}, Z_{s}, Y_{s+\delta_{1}(s)}, Z_{s+\zeta(s)}\right)-f^{\prime}\left(s, Y_{s}, Z_{s}, Y_{s+\delta_{1}(s)}, Z_{s+\zeta(s)}\right)\right|^{2} d s \\
& +\int_{0}^{T} e^{\mu A_{s}}\left\|g\left(s, Y_{s}, Z_{s}, Y_{s+\delta_{3}(s)}, Z_{s+\bar{\zeta}(s)}\right)-g^{\prime}\left(s, Y_{s}, Z_{s}, Y_{s+\delta_{3}(s)}, Z_{s+\bar{\zeta}(s)}\right)\right\|^{2} d s \\
& \left.+\int_{0}^{T} e^{\mu A_{s}}\left|h\left(s, Y_{s}, Y_{s+\delta_{2}(s)}\right)-h^{\prime}\left(s, Y_{s}, Y_{s+\delta_{2}(s)}\right)\right|^{2} d K_{s}^{\prime}\right]
\end{aligned}
$$

where $A_{t}:=\|\bar{K}\|_{t}+K_{t}^{\prime},\|\bar{K}\|_{t}$ is the total variation for process $\bar{K}$ on the interval $[0, t]$.

Proof. Similar to Proposition 1, we assume $K_{T+K}$ is bounded random variable. From Itô's formula, we have:

$$
\begin{aligned}
& e^{\mu A_{t}}\left|\bar{Y}_{t}\right|^{2}+\int_{t}^{T} e^{\mu A_{s}}\left\|\bar{Z}_{s}\right\|^{2} d s+\mu \int_{t}^{T} e^{\mu A_{s}}\left|\bar{Y}_{s}\right|^{2} d A_{s}=e^{\mu K_{T}}\left|\bar{\xi}_{T}\right|^{2}-2 \int_{t}^{T} e^{\mu A_{s}}\left\langle\bar{Y}_{s}, \bar{Z}_{s} \mathrm{~d} W_{s}\right\rangle \\
& +2 \int_{t}^{T} e^{\mu A_{s}}\left\langle\bar{Y}_{s}, f\left(s, Y_{s}, Z_{s}, Y_{s+\delta_{1}(s)}, Z_{s+\zeta(s)}\right)-f^{\prime}\left(s, Y_{s}^{\prime}, Z_{s}^{\prime}, Y_{s+\delta_{1}(s)}^{\prime}, Z_{s+\zeta(s)}^{\prime}\right)\right\rangle d s \\
& +2 \int_{t}^{T} e^{\mu A_{s}}\left\langle\bar{Y}_{s}, h\left(s, Y_{s}, Y_{s+\delta_{2}(s)}\right)\right\rangle d \bar{K}_{s}+2 \int_{t}^{T} e^{\mu A_{s}}\left\langle\bar{Y}_{s}, h\left(s, Y_{s}, Y_{s+\delta_{2}(s)}\right)-h^{\prime}\left(s, Y_{s}^{\prime}, Y_{s+\delta_{2}(s)}^{\prime}\right)\right\rangle d K_{s}^{\prime} \\
& +2 \int_{t}^{T} e^{\mu A_{s}}\left\langle\bar{Y}_{s},\left(g\left(s, Y_{s}, Z_{s}, Y_{s+\delta_{3}(s)}, Z_{s+\bar{\zeta}(s)}\right)-g^{\prime}\left(s, Y_{s}^{\prime}, Z_{s}^{\prime}, Y_{s+\delta_{3}(s)}^{\prime}, Z_{s+\bar{\zeta}(s)}^{\prime}\right)\right) \mathrm{d} \overleftarrow{B}_{s}\right\rangle \\
& +\int_{t}^{T} e^{\mu A_{s}}\left\|g\left(s, Y_{s}, Z_{s}, Y_{s+\delta_{3}(s)}, Z_{s+\bar{\zeta}(s)}\right)-g^{\prime}\left(s, Y_{s}^{\prime}, Z_{s}^{\prime}, Y_{s+\delta_{3}(s)}^{\prime}, Z_{s+\bar{\zeta}(s)}^{\prime}\right)\right\|^{2} d s
\end{aligned}
$$




$$
\begin{aligned}
& =e^{\mu K_{T}}\left|\bar{\zeta}_{T}\right|^{2}-2 \int_{t}^{T} e^{\mu A_{s}}\left\langle\bar{Y}_{s}, \bar{Z}_{s} \mathrm{~d} W_{s}\right\rangle+2 \int_{t}^{T} e^{\mu A_{s}}\left\langle\bar{Y}_{s}, h\left(s, Y_{s}, Y_{s+\delta_{2}(s)}\right)\right\rangle d \bar{K}_{s} \\
& +2 \int_{t}^{T} e^{\mu A_{s}}\left\langle\bar{Y}_{s}, f\left(s, Y_{s}, Z_{s}, Y_{s+\delta_{1}(s)}, Z_{s+\zeta(s)}\right)-f^{\prime}\left(s, Y_{s}, Z_{s}, Y_{s+\delta_{1}(s)}, Z_{s+\zeta(s)}\right)\right\rangle d s \\
& +2 \int_{t}^{T} e^{\mu A_{s}}\left\langle\bar{Y}_{s}, f^{\prime}\left(s, Y_{s}, Z_{s}, Y_{s+\delta_{1}(s)}, Z_{s+\zeta(s)}\right)-f^{\prime}\left(s, Y_{s}^{\prime}, Z_{s}^{\prime}, Y_{s+\delta_{1}(s)}^{\prime}, Z_{s+\zeta(s)}^{\prime}\right)\right\rangle d s \\
& +2 \int_{t}^{T} e^{\mu A_{s}}\left\langle\bar{Y}_{s}, h\left(s, Y_{s}, Y_{s+\delta_{2}(s)}\right)-h^{\prime}\left(s, Y_{s}, Y_{s+\delta_{2}(s)}\right)\right\rangle d K_{s}^{\prime} \\
& +2 \int_{t}^{T} e^{\mu A_{s}}\left\langle\bar{Y}_{s}, h^{\prime}\left(s, Y_{s}, Y_{s+\delta_{2}(s)}\right)-h^{\prime}\left(s, Y_{s}^{\prime}, Y_{s+\delta_{2}(s)}^{\prime}\right)\right\rangle d K_{s}^{\prime} \\
& +2 \int_{t}^{T} e^{\mu A_{s}}\left\langle\bar{Y}_{s},\left(g\left(s, Y_{s}, Z_{s}, Y_{s+\delta_{3}(s)}, Z_{s+\bar{\zeta}(s)}\right)-g^{\prime}\left(s, Y_{s}^{\prime}, Z_{s}^{\prime}, Y_{s+\delta_{3}(s)}^{\prime}, Z_{s+\bar{\zeta}(s)}^{\prime}\right)\right) \mathrm{d} \overleftarrow{B}_{s}\right\rangle \\
& +\int_{t}^{T} e^{\mu A_{s}}\left\|g\left(s, Y_{s}, Z_{s}, Y_{s+\delta_{3}(s)}, Z_{s+\bar{\zeta}(s)}\right)-g^{\prime}\left(s, Y_{s}^{\prime}, Z_{s}^{\prime}, Y_{s+\delta_{3}(s)}^{\prime}, Z_{s+\bar{\zeta}(s)}^{\prime}\right)\right\|^{2} d s .
\end{aligned}
$$

Through the assumptions (A1), (A2), (H2) and Yong's inequality, for any $\theta>0$, we get

$$
\begin{aligned}
& \mathbb{E}\left[e^{\mu A_{t}}\left|\bar{Y}_{t}\right|^{2}+\int_{t}^{T} e^{\mu A_{s}}\left\|\bar{Z}_{s}\right\|^{2} d s+\mu \int_{t}^{T} e^{\mu A_{s}}\left|\bar{Y}_{s}\right|^{2} d A_{s}\right] \\
& \leq \mathbb{E}\left[e^{\mu K_{T}}\left|\bar{\xi}_{T}\right|^{2}+\mu \int_{t}^{T} e^{\mu A_{s}}\left|\bar{Y}_{s}\right|^{2} d\|\bar{K}\|_{s}+\frac{1}{\mu} \int_{t}^{T} e^{\mu A_{s}}\left|h\left(s, Y_{s}, Y_{s+\delta_{2}(s)}\right)\right|^{2} d\|\bar{K}\|_{s}\right. \\
& +\int_{t}^{T} e^{\mu A_{s}}\left|\bar{Y}_{s}\right|^{2} d s+\int_{t}^{T} e^{\mu A_{s}}\left|f\left(s, Y_{s}, Z_{s}, Y_{s+\delta_{1}(s)}, Z_{s+\zeta(s)}\right)-f^{\prime}\left(s, Y_{s}, Z_{s}, Y_{s+\delta_{1}(s)}, Z_{s+\zeta(s)}\right)\right|^{2} d s \\
& +\frac{4(M+1) C}{1-\alpha_{1}-\alpha_{2} M} \int_{t}^{T} e^{\mu A_{s}}\left|\bar{Y}_{s}\right|^{2} d s+\frac{1-\alpha_{1}-\alpha_{2} M}{4(M+1)}\left(\int_{t}^{T} e^{\mu A_{s}}\left|\bar{Y}_{s}\right|^{2} d s+\int_{t}^{T} e^{\mu A_{s}}\left\|\bar{Z}_{s}\right\|^{2} d s\right. \\
& \left.+\int_{t}^{T} e^{\mu A_{s} \mathbb{E}^{\mathscr{F}_{s}}}\left|\bar{Y}_{s+\delta_{1}(s)}\right|^{2} d s+\int_{t}^{T} e^{\mu A_{s}} \mathbb{E}^{\mathscr{F}_{s}}|| \bar{Z}_{s+\zeta(s)} \|^{2} d s\right)+\theta \int_{t}^{T} e^{\mu A_{s}}\left|\bar{Y}_{s}\right|^{2} d K_{s}^{\prime} \\
& +\frac{1}{\theta} \int_{t}^{T} e^{\mu A_{s}}\left|h\left(s, Y_{s}, Y_{s+\delta_{2}(s)}\right)-h^{\prime}\left(s, Y_{s}, Y_{s+\delta_{2}(s)}\right)\right|^{2} d K_{s}^{\prime} \\
& +(2 \bar{C}+\beta) \int_{t}^{T} e^{\mu A_{s}}\left|\bar{Y}_{s}\right|^{2} d K_{s}^{\prime}+\beta \int_{t}^{T} e^{\mu A_{s}} \mathbb{E}^{\mathscr{F}_{s}}\left|\bar{Y}_{s+\delta_{2}(s)}\right|^{2} d K_{s}^{\prime} \\
& +\left(1+\frac{2\left(\alpha_{1}+\alpha_{2} M\right)}{1-\alpha_{1}-\alpha_{2} M}\right) \int_{t}^{T} e^{\mu A_{s}}\left\|g\left(s, Y_{s}, Z_{s}, Y_{s+\delta_{3}(s)}, Z_{s+\bar{\zeta}(s)}\right)-g^{\prime}\left(s, Y_{s}, Z_{s}, Y_{s+\delta_{3}(s)}, Z_{s+\bar{\zeta}(s)}\right)\right\|^{2} d s \\
& +\left(1+\frac{1-\alpha_{1}-\alpha_{2} M}{2\left(\alpha_{1}+\alpha_{2} M\right)}\right)\left(C\left(\int_{t}^{T} e^{\mu A_{s}}\left|\bar{Y}_{s}\right|^{2} d s+\int_{t}^{T} e^{\mu A_{s}} \mathbb{E}^{\mathscr{F}_{s}}\left|\bar{Y}_{s+\delta_{3}(s)}\right|^{2} d s\right)\right. \\
& \left.\left.+\alpha_{1} \int_{t}^{T} e^{\mu A_{s}}\left\|\bar{Z}_{s}\right\|^{2} d s+\alpha_{2} \int_{t}^{T} e^{\mu A_{s}} \mathbb{E}^{\mathscr{F}_{s}}\left\|\bar{Z}_{s+\bar{\zeta}(s)}\right\|^{2} d s\right)\right] .
\end{aligned}
$$

Choosing $\theta=\mu-|2 \bar{C}+\beta(1+M)|$, we have:

$\mathbb{E}\left[e^{\mu A_{t}}\left|\bar{Y}_{t}\right|^{2}+\frac{1-\alpha_{1}-\alpha_{2} M}{4} \int_{t}^{T} e^{\mu A_{s}}\left\|\bar{Z}_{s}\right\|^{2} d s\right] \leq \mathbb{E}\left[e^{\mu K_{T}}\left|\bar{\xi}_{T}\right|^{2}+\frac{1}{\mu} \int_{t}^{T} e^{\mu A_{s}}\left|h\left(s, Y_{s}, Y_{s+\delta_{2}(s)}\right)\right|^{2} d\|\bar{K}\|_{s}\right.$

$$
\begin{aligned}
& +\int_{t}^{T} e^{\mu A_{s}}\left|f\left(s, Y_{s}, Z_{s}, Y_{s+\delta_{1}(s)}, Z_{s+\zeta(s)}\right)-f^{\prime}\left(s, Y_{s}, Z_{s}, Y_{s+\delta_{1}(s)}, Z_{s+\zeta(s)}\right)\right|^{2} d s \\
& +\left(\frac{5-\alpha_{1}-\alpha_{2} M}{4}+\frac{4(M+1) C}{1-\alpha_{1}-\alpha_{2} M}+\frac{1+\alpha_{1}+\alpha_{2} M}{2\left(\alpha_{1}+\alpha_{2} M\right)} C(1+M)\right) \int_{t}^{T} e^{\mu A_{s}}\left|\bar{Y}_{s}\right|^{2} d s \\
& +\left(\frac{1+\alpha_{1}+\alpha_{2} M}{2\left(\alpha_{1}+\alpha_{2} M\right)} C+\frac{1-\alpha_{1}-\alpha_{2} M}{4(M+1)}\right) M \int_{T}^{T+K} e^{\mu A_{s}}\left|\bar{\zeta}_{s}\right|^{2} d s \\
& +\left(\frac{1+\alpha_{1}+\alpha_{2} M}{2\left(\alpha_{1}+\alpha_{2} M\right)} \alpha_{2}+\frac{1-\alpha_{1}-\alpha_{2} M}{4(M+1)}\right) M \int_{T}^{T+K} e^{\mu A_{s}}|| \bar{\eta}_{s} \|^{2} d s+\beta M \int_{T}^{T+K} e^{\mu A_{s}}\left|\bar{\xi}_{s}\right|^{2} d K_{s}^{\prime} \\
& +\frac{1}{\mu-|2 \bar{C}+\beta(1+M)|} \int_{t}^{T} e^{\mu A_{s}}\left|h\left(s, Y_{s}, Y_{s+\delta_{2}(s)}\right)-h^{\prime}\left(s, Y_{s}, Y_{s+\delta_{2}(s)}\right)\right|^{2} d K_{s}^{\prime}
\end{aligned}
$$


$\left.+\frac{1+\alpha_{1}+\alpha_{2} M}{1-\alpha_{1}-\alpha_{2} M} \int_{t}^{T} e^{\mu A_{s}}\left\|g\left(s, Y_{s}, Z_{s}, Y_{s+\delta_{3}(s)}, Z_{s+\bar{\zeta}(s)}\right)-g^{\prime}\left(s, Y_{s}, Z_{s}, Y_{s+\delta_{3}(s)}, Z_{s+\bar{\zeta}(s)}\right)\right\|^{2} d s\right]$.

From Gronwall's lemma, we get:

$$
\begin{aligned}
& \sup _{t \in[0, T]} \mathbb{E}\left[e^{\mu A_{t}}\left|\bar{Y}_{t}\right|^{2}\right]+\mathbb{E}\left[\int_{0}^{T} e^{\mu A_{s}}\left\|\bar{Z}_{s}\right\|^{2} d s\right] \leq \hat{C} \mathbb{E}\left[e^{\mu K_{T}}\left|\bar{\xi}_{T}\right|^{2}+\int_{0}^{T} e^{\mu A_{s}}\left|h\left(s, Y_{s}, Y_{s+\delta_{2}(s)}\right)\right|^{2} d\|\bar{K}\|_{s}\right. \\
& +\int_{0}^{T} e^{\mu A_{s}}\left|f\left(s, Y_{s}, Z_{s}, Y_{s+\delta_{1}(s)}, Z_{s+\zeta(s)}\right)-f^{\prime}\left(s, Y_{s}, Z_{s}, Y_{s+\delta_{1}(s)}, Z_{s+\zeta(s)}\right)\right|^{2} d s \\
& +\int_{T}^{T+K} e^{\mu A_{s}}\left|\bar{\xi}_{s}\right|^{2} d s+\int_{T}^{T+K} e^{\mu A_{s}}\left\|\bar{\eta}_{s}\right\|^{2} d s+\int_{T}^{T+K} e^{\mu A_{s}}\left|\bar{\xi}_{s}\right|^{2} d K_{s}^{\prime} \\
& +\int_{0}^{T} e^{\mu A_{s}}\left|h\left(s, Y_{s}, Y_{s+\delta_{2}(s)}\right)-h^{\prime}\left(s, Y_{s}, Y_{s+\delta_{2}(s)}\right)\right|^{2} d K_{s}^{\prime} \\
& \left.+\int_{0}^{T} e^{\mu A_{s}}\left\|g\left(s, Y_{s}, Z_{s}, Y_{s+\delta_{3}(s)}, Z_{s+\bar{\zeta}(s)}\right)-g^{\prime}\left(s, Y_{s}, Z_{s}, Y_{s+\delta_{3}(s)}, Z_{s+\bar{\zeta}(s)}\right)\right\|^{2} d s\right] .
\end{aligned}
$$

Using the Burkholder-Davis-Gundy's and above inequality, the desired result follows.

With the help of Propositions 1 and 2, we can establish the following existence and uniqueness theorem in this part.

Theorem 1. Assume that (A1), (A2), (H1), (H2) and (H3) hold. Then, AGDDSDE (2) admits a unique solution $(Y, Z) \in \mathcal{S}^{2}\left([0, T+K], \mathbb{R}^{k}\right) \times \mathcal{M}^{2}\left(0, T+K, \mathbb{R}^{k \times d}\right)$.

Proof. The uniqueness is easily given by Proposition 2. We now turn to prove its existence. For $\mu \geq 0$, let $M_{\mu}^{2}(K)$ represent the set of progressively measurable processes $\{X(t), 0 \leq$ $t \leq T+K\}$, which satisfy:

$$
\mathbb{E} \int_{0}^{T+K} e^{\mu K_{t}}\left|X_{t}\right|^{2} d t+\mathbb{E} \int_{0}^{T+K} e^{\mu K_{t}} d K_{t}<\infty,
$$

and $M_{\mu}^{2}$ represents the space of progressively measurable processes $\{X(t), 0 \leq t \leq T+K\}$ which are such that:

$$
\mathbb{E} \int_{0}^{T+K} e^{\mu K_{t}}\left|X_{t}\right|^{2} d t<\infty
$$

We define

$$
\mathscr{B}_{\mu}^{2}=\left(M_{\mu}^{2}(K)\right)^{n} \times\left(M_{\mu}^{2}\right)^{n \times d} .
$$

Giving $(U, V) \in \mathscr{B}_{\mu}^{2}$, by Theorem 2.1 in [9], we can define a map $\Phi$ from $\mathscr{B}_{\mu}^{2}$ to $\mathscr{B}_{\mu}^{2}$ through the equation:

$$
\left\{\begin{aligned}
Y_{t}= & \xi_{T}+\int_{t}^{T} f\left(s, Y_{s}, Z_{s}, U_{s+\delta_{1}(s)}, V_{s+\zeta(s)}\right) \mathrm{d} s+\int_{t}^{T} h\left(s, Y_{s}, U_{s+\delta_{2}(s)}\right) d K_{s} \\
& +\int_{t}^{T} g\left(s, Y_{s}, Z_{s}, U_{s+\delta_{3}(s)}, V_{s+\bar{\zeta}(s)}\right) \mathrm{d} \overleftarrow{B}_{s}-\int_{t}^{T} Z_{s} \mathrm{~d} W_{s}, \quad t \in[0, T], \\
Y_{t}= & \xi_{t}, \quad Z_{t}=\eta_{t}, \quad t \in[T, T+K] .
\end{aligned}\right.
$$

In the following, we will use the Banach contraction principle to prove the existence. Let $(U, V),\left(U^{\prime}, V^{\prime}\right) \in \mathscr{B}_{\mu}^{2},(Y, Z)=\Phi(U, V),\left(Y^{\prime}, Z^{\prime}\right)=\Phi\left(U^{\prime}, V^{\prime}\right)$,

$$
\begin{aligned}
& \bar{U}=U-U^{\prime}, \bar{V}=V-V^{\prime}, \bar{Y}=Y-Y^{\prime}, \bar{Z}=Z-Z^{\prime}, \\
& \bar{f}(s)=f\left(s, Y_{s}, Z_{s}, U_{s+\delta_{1}(s)}, V_{s+\zeta(s)}\right)-f\left(s, Y_{s}^{\prime}, Z_{s}^{\prime}, U_{s+\delta_{1}(s)}^{\prime}, V_{s+\zeta(s)}^{\prime}\right), \\
& \bar{g}(s)=g\left(s, Y_{s}, Z_{s}, U_{s+\delta_{3}(s)}, V_{s+\bar{\zeta}(s)}\right)-g\left(s, Y_{s}^{\prime}, Z_{s}^{\prime}, U_{s+\delta_{3}(s)}^{\prime}, V_{s+\bar{\zeta}(s)}^{\prime}\right) \\
& \bar{h}(s)=h\left(s, Y_{s}, U_{s+\delta_{2}(s)}\right)-h\left(s, Y_{s}^{\prime}, U_{s+\delta_{2}(s)}^{\prime}\right) .
\end{aligned}
$$


Consider the following equations:

$$
\left\{\begin{array}{l}
\bar{Y}_{t}=\int_{t}^{T} \bar{f}(s) \mathrm{d} s+\int_{t}^{T} \bar{h}(s) d K_{s}+\int_{t}^{T} \bar{g}(s) \mathrm{d} \overleftarrow{B}_{s}-\int_{t}^{T} \bar{Z}_{s} \mathrm{~d} W_{s}, t \in[0, T] \\
Y_{t}=0, \quad Z_{t}=0, \quad t \in[T, T+K] .
\end{array}\right.
$$

For any $\lambda, \theta>0$, in view of Itô's formula, we have:

$$
\begin{aligned}
& \mathbb{E}\left[e^{\lambda t+\mu K_{t}}\left|\bar{Y}_{t}\right|^{2}+\lambda \int_{t}^{T} e^{\lambda s+\mu K_{s}}\left|\bar{Y}_{s}\right|^{2} d s+\mu \int_{t}^{T} e^{\lambda s+\mu K_{s}}\left|\bar{Y}_{s}\right|^{2} d K_{s}+\int_{t}^{T} e^{\lambda s+\mu K_{s}}\left\|\bar{Z}_{s}\right\|^{2} d s\right] \\
& =2 \mathbb{E} \int_{t}^{T} e^{\lambda s+\mu K_{s}}\left\langle\bar{Y}_{s}, \bar{f}(s)\right\rangle d s+2 \mathbb{E} \int_{t}^{T} e^{\lambda s+\mu K_{s}}\left\langle\bar{Y}_{s}, \bar{h}(s)\right\rangle d K_{s}+\mathbb{E} \int_{t}^{T} e^{\lambda s+\mu K_{s}}\|\bar{g}(s)\|^{2} d s \\
& \leq\left(\theta+\frac{C}{\theta}+C\right) \mathbb{E} \int_{t}^{T} e^{\lambda s+\mu K_{s}}\left|\bar{Y}_{s}\right|^{2} d s+\left(\frac{C}{\theta}+\alpha_{1}\right) \mathbb{E} \int_{t}^{T} e^{\lambda s+\mu K_{s}}\left\|\bar{Z}_{s}\right\|^{2} d s \\
& +\left(\frac{C M}{\theta}+C M\right) \mathbb{E} \int_{t}^{T} e^{\lambda s+\mu K_{s}}\left|\bar{U}_{s}\right|^{2} d s+\left(\frac{C M}{\theta}+\alpha_{2} M\right) \mathbb{E} \int_{t}^{T} e^{\lambda s+\mu K_{s}}\left\|\bar{V}_{s}\right\|^{2} d s \\
& +(2 \bar{C}+\beta \theta) \mathbb{E} \int_{t}^{T} e^{\lambda s+\mu K_{s}}\left|\bar{Y}_{s}\right|^{2} d K_{s}+\frac{\beta M}{\theta} \mathbb{E} \int_{t}^{T} e^{\lambda s+\mu K_{s}}\left|\bar{U}_{s}\right|^{2} d K_{s}
\end{aligned}
$$

Choosing $\theta=16 \frac{C(M+1)}{1-\alpha_{1}-\alpha_{2} M}+2 \beta M, \lambda=\theta+\frac{C}{\theta}+C+2\left(\frac{C M}{\theta}+C M\right)+2, \mu=|2 \bar{C}+\beta \theta|+$ $2\left(\frac{C M}{\theta}+C M\right)+4$, we have

$$
\begin{aligned}
& \mathbb{E} \int_{t}^{T} e^{\lambda s+\mu K_{s}}\left|\bar{Y}_{s}\right|^{2} d s+\mathbb{E} \int_{t}^{T} e^{\lambda s+\mu K_{s}}\left|\bar{Y}_{s}\right|^{2} d K_{s}+\mathbb{E} \int_{t}^{T}\left(\frac{C}{\theta}+\alpha_{1}\right) e^{\lambda s+\mu K_{s}}\left\|\bar{Z}_{s}\right\|^{2} d s \\
& \leq \frac{1}{2} \mathbb{E} \int_{t}^{T} e^{\lambda s+\mu K_{s}}\left|\bar{Y}_{s}\right|^{2} d s+\frac{1}{2} \mathbb{E} \int_{t}^{T} e^{\lambda s+\mu K_{s}}\left|\bar{Y}_{s}\right|^{2} d K_{s}+\frac{1}{2} \mathbb{E} \int_{t}^{T}\left(\frac{C}{\theta}+\alpha_{1}\right) e^{\lambda s+\mu K_{s}}\left\|\bar{Z}_{s}\right\|^{2} d s .
\end{aligned}
$$

Thus, $\Phi$ is a strict contraction on $\mathscr{B}_{\mu}^{2}$ equipped with the norm

$$
\|(Y, Z)\|_{\lambda, \mu}^{2}=\mathbb{E}\left[\int_{0}^{T} e^{\lambda s+\mu K_{s}}\left|Y_{s}\right|^{2} d s+\int_{0}^{T} e^{\lambda s+\mu K_{s}}\left|Y_{s}\right|^{2} d K_{s}+\int_{0}^{T}\left(\frac{C}{\theta}+\alpha_{1}\right) e^{\lambda s+\mu K_{s}}\left\|Z_{s}\right\|^{2} d s\right] .
$$

The proof of existence is complete.

\section{Comparison Theorems}

In this section, we consider one dimensional AGBDSDEs, that is, $k=1$. Let us first give a comparison theorem of GBDSDEs, which will play a key role in what follows. Assume that, for $i=1,2, \xi^{i} \in L^{2}\left(\mathcal{F}_{T} ; \mathbb{R}\right)$ and $f^{i}(t, y, z):[0, T] \times \Omega \times \mathbb{R} \times \mathbb{R}^{d} \rightarrow \mathbb{R}$ satisfies (H1) and (H3). Then, according to Theorem 2.1 in [9], the following GBDSDE,

$$
Y_{t}^{i}=\xi^{i}+\int_{t}^{T} f^{i}\left(s, Y_{s}^{i}, Z_{s}^{i}\right) \mathrm{d} s+\int_{t}^{T} h\left(s, Y_{s}\right) d K_{s}+\int_{t}^{T} g^{i}\left(s, Y_{s}^{i}, Z_{s}^{i}\right) \mathrm{d} \overleftarrow{B}_{s}-\int_{t}^{T} Z_{s}^{i} \mathrm{~d} W_{s}, \quad t \in[0, T]
$$

admits a unique solution $\left(Y^{i}, Z_{\text {. }}^{i}\right) \in \mathcal{S}^{2}\left([0, T] ; \mathbb{R}^{n}\right) \times \mathcal{M}^{2}\left(0, T ; \mathbb{R}^{n \times d}\right)$ for $i=1,2$. We can assert the following comparison theorem, which generalizes the Theorem 2.2 in [11].

Lemma 1. Let $\left(Y^{1}, Z^{1}\right)$ and $\left(Y^{2}, Z^{2}\right)$ be solutions of GBDSDEs (3) respectively. We suppose that (1) $\xi_{t}^{1} \geqslant \xi_{t}^{2}$, a.s.; (2) $f^{1}\left(t, Y_{t}^{1}, Z_{t}^{1}\right) \geqslant f^{2}\left(t, Y_{t}^{1}, Y_{t}^{1}\right)$ or $f^{1}\left(t, Y_{t}^{2}, Z_{t}^{2}\right) \geqslant f^{2}\left(t, Y_{t}^{2}, Y_{t}^{2}\right)$, a.s., a.e. $t \in[0, T] ;(3) h^{1}\left(t, Y_{t}^{1}\right) \geq h^{2}\left(t, Y_{t}^{1}\right)$ or $h^{1}\left(t, Y_{t}^{2}\right) \geq h^{2}\left(t, Y_{t}^{2}\right)$, a.s., a.e. $t \in[0, T] ;(4) g^{1}$ $\left(t, Y_{t}^{1}, Z_{t}^{1}\right)=g^{2}\left(t, Y_{t}^{1}, Y_{t}^{1}\right)$ or $g^{1}\left(t, Y_{t}^{2}, Z_{t}^{2}\right)=g^{2}\left(t, Y_{t}^{2}, Y_{t}^{2}\right)$, a.s., a.e. $t \in[0, T]$. Then, $Y_{t}^{1} \geqslant$ $Y_{t}^{2}$, a.s., for all $t \in[0, T]$.

Proof. Without loss of generality, we assume that $\xi^{1} \geqslant \xi^{2}$, a.s., and $f^{1}\left(t, Y_{t}^{1}, Z_{t}^{1}\right) \geqslant$ $f^{2}\left(t, Y_{t}^{1}, Z_{t}^{1}\right), h^{1}\left(t, Y_{t}^{1}\right) \geq h^{2}\left(t, Y_{t}^{1}\right)$ a.s., for all $t \in[0, T]$. Denote 


$$
\begin{aligned}
& \hat{Y}_{t}=Y_{t}^{2}-Y_{t}^{1}, \hat{Z}_{t}=Z_{t}^{2}-Z_{t}^{1}, \hat{\xi}=\xi^{2}-\xi^{1} . \\
& \hat{f}_{t}=f^{2}\left(t, Y_{t}^{2}, Z_{t}^{2}\right)-f^{1}\left(t, Y_{t}^{1}, Z_{t}^{1}\right), \hat{g}_{t}=g^{2}\left(t, Y_{t}^{2}, Z_{t}^{2}\right)-g^{1}\left(t, Y_{t}^{1}, Z_{t}^{1}\right), \hat{h}_{t}=h^{2}\left(t, Y_{t}^{2}\right)-h^{1}\left(t, Y_{t}^{1}\right) .
\end{aligned}
$$

Using Itô-Meyer's formula and $\xi^{1} \geqslant \xi^{2}$, a.s., we have:

$$
\mathbb{E}\left|\hat{Y}_{t}^{+}\right|^{2}+\mathbb{E} \int_{t}^{T} 1_{\left\{\hat{Y}_{s} \geq 0\right\}}\left|\hat{Z}_{s}\right|^{2} d s=2 \mathbb{E} \int_{t}^{T} \hat{Y}_{s}^{+} \hat{f}_{s} d s+2 \mathbb{E} \int_{t}^{T} \hat{Y}_{s}^{+} \hat{h}_{s} d K_{s}+\mathbb{E} \int_{t}^{T} 1_{\left\{\hat{Y}_{s} \geq 0\right\}}\left|\hat{g}_{s}\right|^{2} d s
$$

In view of (H3), Young's inequality $2 a b \leq \frac{1}{\theta} a^{2}+\theta b^{2}$ and Jensen's inequality, for any $\theta>0$, we have:

$$
\begin{aligned}
2 \mathbb{E} \int_{t}^{T} \hat{Y}_{s}^{+} \hat{f}_{s} d s & \leq 2 \mathbb{E} \int_{t}^{T} \hat{Y}_{s}^{+}\left(f^{2}\left(s, Y_{s}^{2}, Z_{s}^{2}\right)-f^{2}\left(t, Y_{s}^{1}, Z_{s}^{1}\right)\right) d s \\
& \leq \frac{2 C}{1-\alpha_{1}} \mathbb{E} \int_{t}^{T}\left|\hat{Y}_{s}^{+}\right|^{2} d s+\frac{1-\alpha_{1}}{2 C} \int_{t}^{T} 1_{\left\{\hat{Y}_{s} \geq 0\right\}}\left|f^{2}\left(s, Y_{s}^{2}, Z_{s}^{2}\right)-f^{2}\left(t, Y_{s}^{1}, Z_{s}^{1}\right)\right|^{2} d s \\
& \leq\left(\frac{2 C}{1-\alpha_{1}}+\frac{1-\alpha_{1}}{2}\right) \mathbb{E} \int_{t}^{T}\left|\hat{Y}_{s}^{+}\right|^{2} d s+\frac{1-\alpha_{1}}{2} \mathbb{E} \int_{t}^{T} 1_{\left\{\hat{Y}_{s} \geq 0\right\}}\left|\hat{Z}_{s}\right|^{2} d s,
\end{aligned}
$$$$
2 \mathbb{E} \int_{t}^{T} \hat{Y}_{s}^{+} \hat{h}_{s} d K_{s} \leq 2 \mathbb{E} \int_{t}^{T} \hat{Y}_{s}^{+}\left(h^{2}\left(s, Y_{s}^{2}\right)-h^{2}\left(t, Y_{s}^{1}\right)\right) d s \leq 2 \bar{C} \mathbb{E} \int_{t}^{T} 1_{\left\{\hat{Y}_{s} \geq 0\right\}}\left|\hat{Y}_{s}\right|^{2} d s,
$$

and

$$
\begin{aligned}
\mathbb{E} \int_{t}^{T} 1_{\left\{\hat{Y}_{s} \geq 0\right\}}\left|\hat{g}_{s}\right|^{2} d s & =\mathbb{E} \int_{t}^{T} 1_{\left\{\hat{Y}_{s} \geq 0\right\}}\left|g^{1}\left(s, Y_{s}^{2}, Z_{s}^{2}\right)-g^{1}\left(t, Y_{s}^{1}, Z_{s}^{1}\right)\right|^{2} d s \\
& \leq C \mathbb{E} \int_{t}^{T}\left|\hat{Y}_{s}^{+}\right|^{2} d s+\alpha_{1} \mathbb{E} \int_{t}^{T} 1_{\left\{\hat{Y}_{s} \geq 0\right\}}\left|\hat{Z}_{s}\right|^{2} d s .
\end{aligned}
$$

Then, thanks to the above inequalities, we obtain:

$$
\mathbb{E}\left|\hat{Y}_{t}^{+}\right|^{2} \leq\left(C+\frac{2 C}{1-\alpha_{1}}+\frac{1-\alpha_{1}}{2}\right) \mathbb{E} \int_{t}^{T}\left|\hat{Y}_{s}^{+}\right|^{2} d s
$$

From the Gronwall's inequality, we can obtain:

$$
\mathbb{E}\left|\hat{Y}_{t}^{+}\right|^{2}=0, \quad \text { for all } t \in[0, T] .
$$

Hence

$$
Y_{t}^{1} \geqslant Y_{t}^{2}, \text { a.s., } \quad \text { for all } t \in[0, T]
$$

Now let us turn to the study of the comparison theorem for anticipated GBDSDEs. For $i=1,2$, we first consider the following anticipated BDSDE:

$$
\left\{\begin{aligned}
Y_{t}^{i}= & \xi_{T}^{i}+\int_{t}^{T} f^{i}\left(s, Y_{s}^{i}, Z_{s}^{i}, Y_{s+\delta_{1}^{i}(s)}^{i}, Z_{s+\zeta^{i}(s)}^{i}\right) \mathrm{d} s+\int_{t}^{T} h^{i}\left(s, Y_{s}^{i}, Y_{s+\delta_{2}^{i}(s)}^{i}\right) d K_{s} \\
& +\int_{t}^{T} g^{i}\left(s, Y_{s}^{i}, Z_{s}^{i}, Y_{s+\delta_{3}^{i}(s)}^{i}, Z_{s+\bar{\zeta}^{i}(s)}^{i}\right) \mathrm{d} \overleftarrow{B}_{s}-\int_{t}^{T} Z_{s}^{i} \mathrm{~d} W_{s}, \quad t \in[0, T], \\
Y_{t}^{i}= & \xi_{t}^{i}, \quad Z_{t}^{i}=\eta_{t}^{i}, \quad t \in[T, T+K] .
\end{aligned}\right.
$$

Let us assume that $\delta^{i}(\cdot), \bar{\delta}^{i}(\cdot), \zeta^{i}(\cdot), \bar{\zeta}^{i}(\cdot)$ satisfy (A1) and (A2), $\xi^{i} \in \mathcal{S}^{2}([T, T+K] ; \mathbb{R})$, $\eta \cdot \in \mathcal{M}^{2}\left(T, T+K ; \mathbb{R}^{d}\right)$, and $\left(f^{i}, g^{i}\right)$ satisfies (H1) and (H3). Then, by Theorem 1 , anticipated GBDSDE (4) admits a unique solution $\left(Y_{.}^{i}, Z^{i}\right) \in \mathcal{S}^{2}([0, T+K] ; \mathbb{R}) \times \mathcal{M}^{2}\left(0, T+K ; \mathbb{R}^{d}\right)$ for $i=1$, 2 . 
Theorem 2. Let $\left(Y^{1}, Z^{1}\right)$ and $\left(Y^{2}, Z^{2}\right)$ be solutions of AGBDSDEs (4) respectively. We suppose that (1) $\xi_{t}^{1} \geqslant \xi_{t}^{2}$, a.s., for all $t \in[T, T+K]$; (2) $f^{1}\left(t, Y_{t}^{1}, Z_{t}^{1}, Y_{t+\delta_{1}^{1}(t)}^{1}, Z_{t+\zeta^{1}(t)}^{1}\right) \geqslant$ $f^{2}\left(t, Y_{t}^{1}, Z_{t}^{1}, Y_{t+\delta_{1}^{2}(t)}^{2}, Z_{t+\zeta^{2}(t)}^{2}\right)$ or $f^{1}\left(t, Y_{t}^{2}, Z_{t}^{2}, Y_{t+\delta_{1}^{1}(t)}^{1}, Z_{t+\zeta^{1}(t)}^{1}\right) \geqslant f^{2}\left(t, Y_{t}^{2}, Y_{t}^{2}, Y_{t+\delta_{1}^{2}(t)}^{2}\right.$ $\left.Z_{t+\zeta^{2}(t)}^{2}\right)$, a.s., a.e. $t \in[0, T] ;(3) h^{1}\left(t, Y_{t}^{1}, Y_{t+\delta_{2}^{1}(t)}^{1}\right) \geqslant h^{2}\left(t, Y_{t}^{1}, Y_{t+\delta_{2}^{2}(t)}^{2}\right)$ or $f^{1}\left(t, Y_{t}^{2}, Y_{t+\delta_{2}^{1}(t)}^{1}\right) \geqslant$ $f^{2}\left(t, Y_{t}^{2}, Y_{t+\delta_{2}^{2}(t)}^{2}\right)$, a.s., a.e. $t \in[0, T] ;(4) g^{1}\left(t, Y_{t}^{1}, Z_{t}^{1}, Y_{t+\bar{\delta}^{1}(t)}^{1}, Z_{t+\bar{\zeta}^{1}(t)}^{1}\right)=g^{2}\left(t, Y_{t}^{1}, Y_{t}^{1}, Y_{t+\bar{\delta}^{2}(t)^{\prime}}^{\prime}\right.$ $\left.Z_{t+\bar{\zeta}^{2}(t)}^{2}\right)$ or $g^{1}\left(t, Y_{t}^{2}, Z_{t}^{2}, Y_{t+\bar{\delta}^{1}(t)}^{1}, Z_{t+\bar{\zeta}^{1}(t)}^{1}\right)=g^{2}\left(t, Y_{t}^{2}, Y_{t}^{2}, Y_{t+\bar{\delta}^{1}(t)}^{1}, Z_{t+\bar{\zeta}^{1}(t)}^{1}\right)$, a.s., a.e. $t \in[0, T]$. Then $Y_{t}^{1} \geqslant Y_{t}^{2}$, a.s., for all $t \in[0, T+K]$.

Proof. For $i=1,2$, denote

$$
\begin{aligned}
& F^{i}(t, y, z)=f^{i}\left(t, y, z, Y_{s+\delta_{1}^{i}(s)}^{i}, Z_{s+\zeta^{i}(s)}^{i}\right), G^{i}(t, y, z)=g^{i}\left(t, y, z, Y_{s+\delta_{3}^{i}(s)}^{i}, Z_{s+\bar{\zeta}^{i}(s)}^{i}\right), \\
& H^{i}(t, y)=h^{i}\left(s, y, Y_{s+\delta_{2}^{i}(s)}^{i}\right),
\end{aligned}
$$

then $\left(Y^{i}, Z^{i}\right)$ is the unique solution of the following GBDSDE,

$$
Y_{t}^{i}=\xi_{T}^{i}+\int_{t}^{T} F^{i}\left(s, Y_{s}^{i}, Z_{s}^{i}\right) \mathrm{d} s+\int_{t}^{T} H^{i}\left(s, Y_{s}^{i}\right) d K_{s}+\int_{t}^{T} G^{i}\left(s, Y_{s}^{i}, Z_{s}^{i}\right) \mathrm{d} \overleftarrow{B}_{s}-\int_{t}^{T} Z_{s}^{i} \mathrm{~d} W_{s}, t \in[0, T]
$$

According to Lemma 1, we can get

$$
Y_{t}^{1} \geqslant Y_{t}^{2} \text {, a.s., for all } t \in[0, T],
$$

which implies

$$
Y_{t}^{1} \geqslant Y_{t}^{2} \text {, a.s., for all } t \in[0, T+K] \text {. }
$$

Let us give an example.

Example 1. Let $f^{1}(t, y, z, \theta(r), \vartheta(\bar{r}))=|y|+|z|+\mathbb{E}^{\mathcal{F}_{t}}[|\theta(r)|+1]+\mathbb{E}^{\mathcal{F}_{t}}[|\cos \vartheta(\bar{r})|], f^{2}(t, y, z$, $\theta(r), \vartheta(\bar{r}))=y-|z|+\mathbb{E}^{\mathcal{F}_{t}}[\sin \theta(r)], g^{1}(t, y, z, \theta(r), \vartheta(\bar{r}))=g^{2}(t, y, z, \theta(r), \vartheta(\bar{r}))=y+$ $|z|, h^{1}(t, y, \theta(r))=|y|+\mathbb{E}^{\mathcal{F}_{t}}[|\arctan \theta(r)|], h^{2}(t, y, \theta(r))=y-1$. Then by Theorem 2, we can obtain $Y_{t}^{1} \geqslant Y_{t}^{2}$, a.s., for all $t \in[0, T+K]$ as long as the assumption (1) of Theorem 2 holds.

Next, we turn to the study of another comparison theorem for anticipated GBDSDEs. For $i=1,2$, we consider the following anticipated GBDSDE:

$$
\left\{\begin{aligned}
Y_{t}^{i}= & \xi_{T}^{i}+\int_{t}^{T} f^{i}\left(s, Y_{s}^{i}, Z_{s}^{i}, Y_{s+\delta_{1}(s)}^{i}, Z_{s+\zeta^{i}(s)}^{i}\right) \mathrm{d} s+\int_{t}^{T} h^{i}\left(s, Y_{s}^{i}, Y_{s+\delta_{2}^{i}(s)}^{i}\right) d K_{s} \\
& +\int_{t}^{T} g^{i}\left(s, Y_{s}^{i}, Z_{s}^{i}, Y_{s+\bar{\delta}_{3}^{i}(s)}^{i}, Z_{s+\bar{\zeta}^{i}(s)}^{i}\right) \mathrm{d} \overleftarrow{B}_{s}-\int_{t}^{T} Z_{s}^{i} \mathrm{~d} W_{s}, \quad t \in[0, T] \\
Y_{t}^{i}= & \xi_{t}^{i}, \quad Z_{t}^{i}=\eta_{t}^{i}, \quad t \in[T, T+K] .
\end{aligned}\right.
$$

We always assume that $\left(\delta(\cdot), \zeta^{i}(\cdot), \bar{\delta}^{i}(\cdot), \bar{\zeta}^{i}(\cdot)\right)$ satisfy (A1) and (A2), $\xi^{i} \cdot \in \mathcal{S}^{2}([T, T+K] ; \mathbb{R})$, $\eta^{i} \in \mathcal{M}^{2}\left(T, T+K ; \mathbb{R}^{d}\right)$ and $\left(f^{i}, g^{i}\right)$ satisfy (H1) and (H3). Then, by Theorem 1 , anticipated $\operatorname{GBDSDE}(5)$ admits a unique solution $\left(Y^{i}, Z^{i}.\right) \in \mathcal{S}^{2}([0, T+K] ; \mathbb{R}) \times \mathcal{M}^{2}\left(0, T+K ; \mathbb{R}^{d}\right)$ for $i=1,2$.

Theorem 3. Let $\left(Y^{1}, Z^{1}\right)$ and $\left(Y^{2}, Z^{2}\right)$ be solutions of AGBDSDEs (5) respectively. We suppose that (1) $\xi_{t}^{1} \geqslant \xi_{t}^{2}$, a.s., for all $t \in[T, T+K] ;(2) f^{1}\left(t, Y_{t}^{1}, Z_{t}^{1}, Y_{t+\delta_{1}(t)}^{1}, Z_{t+\zeta^{1}(t)}^{1}\right) \geqslant$ $f^{2}\left(t, Y_{t}^{1}, Z_{t}^{1}, Y_{t+\delta_{1}(t)}^{1}, Z_{t+\zeta^{2}(t)}^{2}\right)$, a.s., a.e.t $\in[0, T]$; (3) $h^{1}\left(t, Y_{t}^{1}, Y_{t+\delta_{2}^{1}(t)}^{1}\right) \geqslant h^{2}\left(t, Y_{t}^{1}, Y_{t+\delta_{2}^{2}(t)}^{2}\right)$, a.s., a.e.t $\in[0, T] ;(4)$ for any $(t, y, z) \in[0, T] \times R \times R^{d}, \gamma \in L^{2}\left(\mathcal{F}_{s} ; \mathbb{R}^{d}\right)$ and $s \in[t, T+$ $K], f^{2}(t, y, z, \cdot \gamma)$ is increasing, that is, $f^{2}\left(t, y, z, y_{2}, \gamma\right) \geq f^{2}\left(t, y, z, y_{1}, \gamma\right)$, if $y_{2} \geq y_{1}$ with 
$y_{1}, y_{2} \in R ;(5) g^{1}\left(t, Y_{t}^{1}, Z_{t}^{1}, Y_{t+\bar{\delta}^{1}(t)}^{1} Z_{t+\bar{\zeta}^{1}(t)}^{1}\right)=g^{2}\left(t, Y_{t}^{1}, Y_{t}^{1}, Y_{t+\bar{\delta}^{2}(t)}^{2}, Z_{t+\bar{\zeta}^{2}(t)}^{2}\right)$, a.s., a.e.t $\in$ $[0, T]$. Then, $Y_{t}^{1} \geqslant Y_{t}^{2}$, a.s., for all $t \in[0, T+K]$.

Proof. For $i=1,2$, denote:

$$
\begin{aligned}
& F^{i}(t, y, z)=f^{i}\left(t, y, z, Y_{t+\delta_{1}(t)}^{i}, Z_{t+\zeta^{i}(t)}^{i}\right), G^{i}(t, y, z)=g^{i}\left(t, y, z, Y_{t+\bar{\delta}_{3}^{i}(t)}^{i}, Z_{t+\bar{\zeta}^{i}(t)}^{i}\right), \\
& H^{i}(t, y)=h^{i}\left(s, y, Y_{s+\delta_{2}^{i}(s)}^{i}\right),
\end{aligned}
$$

then $\left(Y^{i}, Z^{i}\right)$ is the unique solution of the following GBDSDEs,

$$
\left\{\begin{aligned}
Y_{t}^{i}= & \xi_{T}^{i}+\int_{t}^{T} F^{i}\left(s, Y_{s}^{i}, Z_{s}^{i}\right) \mathrm{d} s+\int_{t}^{T} H^{i}\left(s, Y_{s}^{i}\right) d K_{s}+\int_{t}^{T} G^{i}\left(s, Y_{s}^{i}, Z_{s}^{i}\right) \mathrm{d} \overleftarrow{B}_{s} \\
& -\int_{t}^{T} Z_{s}^{i} \mathrm{~d} W_{s}, \quad t \in[0, T], \\
Y_{t}^{i}= & \xi_{t}^{i}, \quad Z_{t}^{i}=\eta_{t}^{i}, \quad t \in[T, T+K] .
\end{aligned}\right.
$$

Let $\bar{f}^{2}(t, y, z)=f^{2}\left(t, y, z, Y_{t+\delta_{1}(t)}^{1}, Z_{t+\zeta^{2}(t)}^{2}\right)$, then the following GBDSDE admits a unique solution $\left(Y^{3}, Z^{3}\right)$,

$$
\left\{\begin{aligned}
Y_{t}^{3}= & \xi_{T}^{2}+\int_{t}^{T} \bar{f}^{2}\left(s, Y_{s}^{3}, Z_{s}^{3}\right) \mathrm{d} s+\int_{t}^{T} H^{2}\left(s, Y_{s}^{3}\right) d K_{s}+\int_{t}^{T} G^{2}\left(s, Y_{s}^{3}, Z_{s}^{3}\right) \mathrm{d} \overleftarrow{B}_{s} \\
& -\int_{t}^{T} Z_{s}^{3} \mathrm{~d} W_{s}, \quad t \in[0, T], \\
Y_{t}^{3}= & \xi_{t}^{2}, \quad Z_{t}^{3}=\eta_{t}^{2}, \quad t \in[T, T+K] .
\end{aligned}\right.
$$

According to the assumptions (1), (2), (4) and Lemma 1, we can get $Y_{t}^{1} \geqslant Y_{t}^{3}$, a.s., for all $\mathrm{t} \in$ $[0, T]$, which implies $Y_{t}^{1} \geqslant Y_{t}^{3}$, a.s., for all $\mathrm{t} \in[0, \mathrm{~T}+\mathrm{K}]$. Let $\left(Y^{4}, Z^{4}\right)$ be the unique solution for the following GBDSDE:

$$
\left\{\begin{aligned}
Y_{t}^{4}= & \xi_{T}^{2}+\int_{t}^{T} f^{2}\left(s, Y_{s}^{4}, Z_{s}^{4}, Y_{t+\delta_{1}(t)}^{3}, Z_{t+\zeta^{2}(t)}^{2}\right) \mathrm{d} s+\int_{t}^{T} H^{2}\left(s, Y_{s}^{4}\right) d K_{s}+\int_{t}^{T} G^{2}\left(s, Y_{s}^{4}, Z_{s}^{4}\right) \mathrm{d} \overleftarrow{B}_{s} \\
& -\int_{t}^{T} Z_{s}^{4} \mathrm{~d} W_{s}, t \in[0, T], \\
Y_{t}^{4}= & \xi_{t}^{2}, \quad Z_{t}^{4}=\eta_{t}^{2}, \quad t \in[T, T+K] .
\end{aligned}\right.
$$

From Lemma 1 and assumptions (3), we can get $Y_{t}^{3} \geqslant Y_{t}^{4}$, a.s., for all $\mathrm{t} \in[0, \mathrm{~T}]$, which implies $Y_{t}^{3} \geqslant Y_{t}^{4}$, a.s., for all $\mathrm{t} \in[0, \mathrm{~T}+\mathrm{K}]$. For $j \geq 5$, define:

$$
\left\{\begin{aligned}
Y_{t}^{j}= & \xi_{T}^{2}+\int_{t}^{T} f^{2}\left(s, Y_{s}^{j}, Z_{s}^{j}, Y_{t+\delta_{1}(t)}^{j-1}, Z_{t+\zeta^{2}(t)}^{2}\right) \mathrm{d} s+\int_{t}^{T} H^{2}\left(s, Y_{s}^{j}\right) d K_{s}+\int_{t}^{T} G^{2}\left(s, Y_{s}^{j}, Z_{s}^{j}\right) \mathrm{d} \overleftarrow{B}_{s} \\
& -\int_{t}^{T} Z_{s}^{j} \mathrm{~d} W_{s}, t \in[0, T], \\
Y_{t}^{j}= & \tau_{t}^{2}, \quad Z_{t}^{j}=\eta_{t}^{2}, \quad t \in[T, T+K] .
\end{aligned}\right.
$$

According to Lemma 1 and by induction, we can get $Y_{t}^{4} \geq Y_{t}^{5} \geq \cdots \geq Y_{t}^{j-1} \geq Y_{t}^{j}$, a.s., for all $t \in[0, T+K]$; hence, for all $j \geq 3$

$$
Y_{t}^{1} \geqslant Y_{t}^{j} \text {,a.s., for all } t \in[0, T+K] .
$$

Set

$$
\hat{Y}_{t}^{j}=Y_{t}^{j}-Y_{t}^{j-1}, \hat{Z}_{t}^{j}=Z_{t}^{j}-Z_{t}^{j-1},
$$




$$
\begin{aligned}
& \hat{F}_{t}^{j}=f^{2}\left(t, Y_{t}^{j}, Z_{t}^{j}, Y_{t+\delta_{1}(t)}^{j-1}, Z_{t+\zeta^{2}(t)}^{2}\right)-f^{2}\left(t, Y_{t}^{j-1}, Z_{t}^{j-1}, Y_{t+\delta_{1}(t)}^{j-2}, Z_{t+\zeta^{2}(t)}^{2}\right), \\
& \hat{G}_{t}^{j}=G^{2}\left(t, Y_{t}^{j}, Z_{t}^{j}\right)-G^{2}\left(t, Y_{t}^{j-1}, Z_{t}^{j-1}\right), \hat{H}_{t}^{j}=H^{2}\left(t, Y_{t}^{j}\right)-H^{2}\left(t, Y_{t}^{j-1}\right) .
\end{aligned}
$$

Then for $j \geq 5,\left(\hat{Y}^{j}, \hat{Z}^{j}\right)$ satisfies

$$
\left\{\begin{array}{l}
\hat{Y}_{t}^{j}=\int_{t}^{T} \hat{F}_{s}^{j} \mathrm{~d} s+\int_{t}^{T} \hat{H}_{s}^{j} d K_{s}+\int_{t}^{T} \hat{G}_{s}^{j} \mathrm{~d} \overleftarrow{B}_{s}-\int_{t}^{T} \hat{Z}_{s}^{j} \mathrm{~d} W_{s}, t \in[0, T] \\
\hat{Y}_{t}^{j}=0, \quad \hat{Z}_{t}^{j}=0, \quad t \in[T, T+K] .
\end{array}\right.
$$

For any $\lambda, \mu, \theta>0$, apply Itô's formula to $e^{\lambda t+\mu K_{t}}\left|\hat{Y}_{t}^{j}\right|^{2}$, in view of (H3), Young's inequality, Jensen's inequality, we have:

$$
\begin{aligned}
& \mathbb{E}\left[e^{\lambda t+\mu K_{t}}\left|\hat{Y}_{t}^{j}\right|^{2}+\lambda \int_{t}^{T} e^{\lambda s+\mu K_{s}}\left|\hat{Y}_{s}^{j}\right|^{2} d s+\mu \int_{t}^{T} e^{\lambda s+\mu K_{s}}\left|\hat{Y}_{s}^{j}\right|^{2} d K_{s}+\int_{t}^{T} e^{\lambda s+\mu K_{s}}\left|\hat{Z}_{s}^{j}\right|^{2} d s\right] \\
& =2 \mathbb{E} \int_{t}^{T} e^{\lambda s+\mu K_{s}} \hat{Y}_{s}^{j} \hat{F}_{s}^{j} d s+2 \mathbb{E} \int_{t}^{T} e^{\lambda s+\mu K_{s}} \hat{Y}_{s}^{j} \hat{H}_{s}^{j} d K_{s}+\mathbb{E} \int_{t}^{T} e^{\lambda s+\mu K_{s}}\left|\hat{G}_{s}^{j}\right|^{2} d s \\
& \leq\left(\theta+\frac{C}{\theta}+C\right) \mathbb{E} \int_{t}^{T} e^{\lambda s+\mu K_{s}}\left|\bar{Y}_{s}\right|^{2} d s+\left(\frac{C}{\theta}+\alpha_{1}\right) \mathbb{E} \int_{t}^{T} e^{\lambda s+\mu K_{s}}\left|\bar{Z}_{s}\right|^{2} d s \\
& +\frac{C M}{\theta} \mathbb{E} \int_{t}^{T} e^{\lambda s+\mu K_{s}}\left|\hat{Y}_{s}^{j-1}\right|^{2} d s+2 \bar{C} \mathbb{E} \int_{t}^{T} e^{\lambda s+\mu K_{s}}\left|\hat{Y}_{s}^{j}\right|^{2} d K_{s} .
\end{aligned}
$$

Choosing $\theta=\frac{2 C}{1-\alpha_{1}}+2 C M, \lambda=\theta+\frac{C}{\theta}+C+1, \mu=1$, we have:

$$
\begin{aligned}
& \mathbb{E}\left[\int_{t}^{T} e^{\lambda s+\mu K_{s}}\left|\hat{Y}_{s}^{j}\right|^{2} d s+\int_{t}^{T} e^{\lambda s+\mu K_{s}}\left|\hat{Y}_{s}^{j}\right|^{2} d K_{s}+\int_{t}^{T}\left(1-\frac{C}{\theta}-\alpha_{1}\right) e^{\lambda s+\mu K_{s}}\left|\hat{Z}_{s}^{j}\right|^{2} d s\right] \\
& \leq \frac{1}{2} \mathbb{E}\left[\int_{t}^{T} e^{\lambda s+\mu K_{s}}\left|\hat{Y}_{s}^{j-1}\right|^{2} d s+\int_{t}^{T} e^{\lambda s+\mu K_{s}}\left|\hat{Y}_{s}^{j-1}\right|^{2} d K_{s}+\int_{t}^{T}\left(1-\frac{C}{\theta}-\alpha_{1}\right) e^{\lambda s+\mu K_{s}}\left|\hat{Z}_{s}^{j-1}\right|^{2} d s\right] .
\end{aligned}
$$

Thus, we have proved that $\left(Y_{.}^{k}, Z_{.}^{k}\right)$ is a Cauchy sequence in $\mathscr{B}_{\mu}^{2}$ with the norm,

$$
\|(Y, Z)\|_{\lambda, \mu}^{2}=\mathbb{E}\left[\int_{0}^{T} e^{\lambda s+\mu K_{s}}\left|Y_{s}\right|^{2} d s+\int_{0}^{T} e^{\lambda s+\mu K_{s}}\left|Y_{s}\right|^{2} d K_{s}+\int_{0}^{T}\left(1-\frac{C}{\theta}-\alpha_{1}\right) e^{\lambda s+\mu K_{s}}\left|Z_{s}\right|^{2} d s\right],
$$

so it is also a Cauchy sequence in $M^{2}(0, T+K ; \mathbb{R}) \times M^{2}\left(0, T+K ; \mathbb{R}^{d}\right)$. Therefore, there exists $(Y ., Z$. $) \in M^{2}(0, T+K ; \mathbb{R}) \times M^{2}\left(0, T+K ; \mathbb{R}^{d}\right)$ such that $Y_{t}=\tilde{\xi}_{t}^{2}, Z_{t}=\eta_{t}^{2}$ for $T \leqslant t \leqslant T+K$ and

$$
\mathbb{E}\left[\int_{0}^{T}\left|Y_{t}^{j}-Y_{t}\right|^{2} \mathrm{~d} K_{t}\right]+\mathbb{E}\left[\int_{0}^{T}\left|Y_{t}^{j}-Y_{t}\right|^{2} \mathrm{~d} t\right]+\mathbb{E}\left[\int_{0}^{T}\left|Z_{t}^{j}-Z_{t}\right|^{2} \mathrm{~d} t\right] \rightarrow 0 \text { as } j \rightarrow \infty .
$$

Hence, it is easy to check that, as $j \rightarrow \infty$,

$$
\begin{gathered}
\mathbb{E} \int_{t}^{T}\left|f^{2}\left(s, Y_{s}^{j}, Z_{s}^{j}, Y_{s+\delta_{1}(s)}^{j-1}, Z_{s+\zeta^{2}(s)}^{2}\right)-f^{2}\left(s, Y_{s}, Z_{s}, Y_{s+\delta(s)}, Z_{s+\zeta^{2}(s)}^{2}\right)\right|^{2} \mathrm{~d} s \rightarrow 0, \\
\mathbb{E} \int_{t}^{T}\left|g^{2}\left(s, Y_{s}^{j}, Z_{s}^{j}, Y_{s+\bar{\delta}^{2}(s)^{\prime}}^{2} Z_{s+\bar{\zeta}^{2}(s)}^{2}\right)-g^{2}\left(s, Y_{s}, Z_{s}, Y_{s+\bar{\delta}^{2}(s)}^{2}, Z_{s+\bar{\zeta}^{2}(s)}^{2}\right)\right|^{2} \mathrm{~d} s \rightarrow 0, \\
\mathbb{E}\left[\left|\int_{t}^{T} Z_{s}^{j} \cdot \mathrm{d} W_{s}-\int_{t}^{T} Z_{s} \cdot \mathrm{d} W_{s}\right|^{2}\right] \rightarrow 0 .
\end{gathered}
$$

Furthermore, we can prove $Y . \in S^{2}(0, T+K ; \mathbb{R})$ through Burkholder-Davis-Gundy inequality. So, we conclude that $(Y ., Z$. $)$ solves the following AGBDSDE: 


$$
\left\{\begin{aligned}
Y_{t}= & \xi_{T}^{2}+\int_{t}^{T} f^{2}\left(s, Y_{s}, Z_{s}, Y_{s+\delta_{1}(s)}, Z_{s+\zeta^{2}(s)}^{2}\right) \mathrm{d} s+\int_{t}^{T} h^{2}\left(s, Y_{s}, Y_{s+\delta_{2}^{2}(s)}^{2}\right) d K_{s} \\
& +\int_{t}^{T} g^{2}\left(s, Y_{s}, Z_{s}, Y_{s+\bar{\delta}_{3}^{2}(s)}^{2}, Z_{s+\bar{\zeta}^{2}(s)}^{2}\right) \mathrm{d} \overleftarrow{B}_{s}-\int_{t}^{T} Z_{s} \mathrm{~d} W_{s}, \quad t \in[0, T] \\
Y_{t}= & \xi_{t}^{2}, \quad Z_{t}=\eta_{t}^{2}, \quad t \in[T, T+K] .
\end{aligned}\right.
$$

Then the uniqueness part of Theorem 1 shows that $Y_{t}=Y_{t}^{2}$, a.s., for all $t \in[0, T+K]$. Finally, letting $j \rightarrow \infty$ in (6) yields $Y_{t}^{1} \geq Y_{t}^{2}$, a.s., for all $t \in[0, T+K]$.

Let us give an example.

Example 2. Let $f^{1}(t, y, z, \theta(r), \vartheta(\bar{r}))=|y|+|z|+\mathbb{E}^{\mathcal{F}_{t}}[|\theta(r)|]+\mathbb{E}^{\mathcal{F}_{t}}[|\cos \vartheta(\bar{r})|], f^{2}(t, y, z$, $\theta(r), \vartheta(\bar{r}))=y+|z|+\mathbb{E}^{\mathcal{F}_{t}}[\theta(r)], g^{1}(t, y, z, \theta(r), \vartheta(\bar{r}))=g^{2}(t, y, z, \theta(r), \vartheta(\bar{r}))=\arctan y+$ $|z|, h^{1}(t, y, \theta(r))=|y|+\mathbb{E}^{\mathcal{F}_{t}}[\arctan \theta(r)], h^{2}(t, y, \theta(r))=y-\frac{\pi}{2}$. Then by Theorem 3 , we can derive $Y_{t}^{1} \geqslant Y_{t}^{2}$, a.s., for all $t \in[0, T+K]$ as long as the assumption (1) of Theorem 3 holds.

\section{Conclusions}

In this paper, we explore a class of anticipated AGBDSDEs. We proved the existence and uniqueness of the solutions of this kind of AGBDSDE. Moreover, two comparison theorems are also proved. In the coming future papers, we will focus on studying this topic and pay more attention to the applications of such equations.

Author Contributions: Writing-original draft preparation, T.W. and J.Y.; writing-review and editing, T.W. and J.Y.; Conceptualization, T.W. All authors have read and agreed to the published version of the manuscript.

Data Availability Statement: Not applicable.

Acknowledgments: The authors express their sincerest thanks to the reviewers for their valuable comments, which further improve the conclusion and proof process of the article.

Conflicts of Interest: The authors declare no conflict of interest.

\section{References}

1. Pardoux, E.; Peng, S. Adaptated solutions of backward stochastic differential equations. Syst. Control Lett. 1990, 14, 535-581. [CrossRef]

2. El Karoui, N.; Peng, S.; Quenez, M.C. Backward stochastic differential equations in finance. Math. Financ. 1997, 7, 1-71. [CrossRef]

3. Shokri, A. An explicit trigonometrically fitted ten-step method with phase-lag of order infinity for the numerical solution of the radial Schrodinger equation. Appl. Comput. Math. 2015, 14, 63-74.

4. Pardoux, E.; Zhang, S. Generalized BSDEs and nonlinear Neumann boundary value problems. Probab. Theory Relat. Fields 1998, 110, 535-558. [CrossRef]

5. Ren, Y.; Xia, N. Generalized Reflected BSDE and an Obstacle Problem for PDEs with a Nonlinear Neumann Boundary Condition. Stoch. Anal. Appl. 2006, 24, 1013-1033.

6. Ren, Y.; Otmani, M. Generalized reflected BSDEs driven by a Levy process and an obstacle problem for PDIEs with a nonlinear Neumann boundary condition. J. Comput. Appl. Math. 2010, 233, 2027-2043. [CrossRef]

7. Pardoux, E.; Peng, S. Backward doubly stochastic differential equations and systemes of quasilinear SPDEs. Probab. Theory Relat. Fields 1994, 98, 209-227. [CrossRef]

8. Shi, Y.; Gu, Y.; Liu, K. Comparison theorems of backward doubly stochastic differential equations and applications. Stoch. Anal. Appl. 2005, 23, 97-110. [CrossRef]

9. Boufoussi, B.; Casteren, J.; Mrhardy, N. Generalized backward doubly stochastic differential equations and SPDEs with nonlinear Neumann boundary conditions. Bernoulli 2007, 13, 423-446. [CrossRef]

10. Hu, L.; Ren, Y. Stochastic PDIEs with nonlinear Neumann boundary conditions and generalized backward doubly stochastic differential equations driven by Levy processes. J. Comput. Appl. Math. 2009, 229, 230-239. [CrossRef]

11. Aman, A.; Mrhardy, N. Obstacle problem for SPDE with nonlinear Neumann boundary condition via reflected generalized backward doubly SDEs. Stat. Probabil. Lett. 2013, 83, 863-874. [CrossRef]

12. Yang, Z.; Peng, S. Anticipated backward stochastic differential equations. Ann. Probab. 2009, 37, 877-902.

13. Zhang, F. Comparison theorems for anticiapted BSDEs with non-Lipschitz coefficients. J. Math. Anal. Appl. 2014, 416, 768-782. [CrossRef]

14. Xu, X. Anticipated Backward doubly Stochastic differential equations. Appl. Math. Comput. 2013, 220, 53-62. [CrossRef] 
15. Zhang, F. Anticipated backward doubly stochastic differential equations. Sci. Sin. Math. 2013, 43, 1223-1236. (In Chinese) [CrossRef]

16. Zhu, Q.; Shi, Y.; Wen, J.; Zhang, H. A Type of Time-Symmetric Stochastic System and Related Games. Symmetry 2021 , 13, 118. [CrossRef]

17. Wu, H.; Wang, W.; Guo, Z. Generalized anticipated backward stochastic differential equations driven by Brownian motion and continuous increasing process. Commun. Stat.-Simul. C 2018, 47, 809-821. 